\title{
Distributed stochastic MPC for systems with parameter uncertainty and disturbances
}

\author{
Li Dai ${ }^{1}$, Yuanqing Xia ${ }^{1 * \dagger}$, Yulong $\mathrm{Gao}^{2}$ and Mark Cannon ${ }^{3}$ \\ ${ }^{1}$ School of Automation, Key Laboratory of Intelligent Control and Decision of Complex Systems, Beijing Institute of \\ Technology, Beijing 100081, China \\ ${ }^{2}$ ACCESS Linnaeus Center, KTH Royal Institute of Technology, SE-10044 Stockholm, Sweden \\ ${ }^{3}$ Department of Engineering Science, University of Oxford, Parks Road, Oxford, OX1 3PJ, United Kingdom
}

\begin{abstract}
SUMMARY
A distributed stochastic model predictive control (DSMPC) algorithm is proposed for multiple linear subsystems with both parameter uncertainty and stochastic disturbances, which are coupled via probabilistic constraints. To handle the probabilistic constraints, the system dynamics is first decomposed into a nominal part and an uncertain part. The uncertain part is further divided into two parts: the first one is constrained to lie in probabilistic tubes that are calculated offline through the use of the probabilistic information on disturbances, whereas the second one is constrained to lie in polytopic tubes whose volumes are optimized online and whose facets' orientations are determined offline. By permitting a single subsystem to optimize at each time step, the probabilistic constraints are then reduced into a set of linear deterministic constraints, and the online optimization problem is transformed into a convex optimization problem that can be performed efficiently. Furthermore, compared to a centralized control scheme, the DSMPC algorithm only requires message transmissions when a subsystem is optimized, thereby offering greater flexibility in communication. By designing a tailored invariant terminal set for each subsystem, the proposed algorithm can achieve recursive feasibility, which in turn ensures closed-loop stability of the entire system. A numerical example is given to illustrate the efficacy of the algorithm. Copyright (c) 2010 John Wiley \& Sons, Ltd.
\end{abstract}

Received ...

KEY WORDS: stochastic systems; probabilistic constraints; model predictive control (MPC); distributed control

\section{INTRODUCTION}

The problem of control and optimization for a team of constrained subsystems subject to both model uncertainty and exogenous influence is an important but rather difficult problem in many practical applications [1, 2, 3], such as formation control of multi-agent systems and investment portfolio optimization. This problem has been tentatively addressed by several control strategies, in which distributed stochastic model predictive control (DSMPC) stands out due to its good control performance, structural flexibility, and capabilities of constraint handling. However, the research of DSMPC is still in an embryonic stage, and lots of challenges still remain to be solved. For example, how to handle the coupling among stochastic subsystems in a distributed fashion; how to transform coupled probabilistic constraints into local deterministic constraints and formulate a tractable optimization problem; how to design distributed controllers which ensure recursive

${ }^{*}$ Correspondence to: Yuanqing Xia, School of Automation, Beijing Institute of Technology, 5 South Zhongguancun Street, Haidian District, Beijing 100081, China.

†xia_yuanqing@bit.edu.cn 
feasibility and closed-loop stability; and how to achieve low frequency of communication and flexibility in communication.

To date, methods accounting for both parameter uncertainty and disturbances have only been developed for single systems $[4,5,6]$. Based on an augmented autonomous state space description in [7], a computationally convenient SMPC approach was investigated in [4]. By using an extension of the concept of invariance, namely invariance with probability, and confining the augmented state to the invariant set with probability, it is possible to achieve satisfaction of constraints with given probabilities and establish recursive feasibility and closed-loop stability. The authors of [4] only considered a particular type of probabilistic constraints which take the form of limits on the expected number of samples at which the system output lies outside a desired interval over a given horizon. In addition, both disturbances and uncertain parameters considered were limited to be independent and identically distributed (i.i.d.) Gaussian random variables. The recent result in [5] further addressed the case of systems with unmeasurable states. An augmented system of both estimated states and estimation errors was formulated and polytopic tubes were used to bound the evolution of the augmented system. The proposed output-feedback SMPC algorithm was shown to be recursively feasible despite the action of uncertainties. However, [5] only proved the convergence of system states to a neighborhood of the origin in simulations and cannot ensure closed-loop stability in theory. Furthermore, the system matrices considered were expressed as affine functions of one scalar uncertain parameter, which limits the applicability of this algorithm. In [6], the state trajectory was decomposed into three parts which were handled separately to ensure probabilistic constraint satisfaction and the resulting algorithm provided desirable closedloop properties: recursive feasibility and stability.

To the best of our knowledge, few existing works on distributed MPC (DMPC) handle multiple subsystems with both parameter uncertainty and stochastic disturbances. Different from single systems, each subsystem in DMPC scheme is required to consider not only the uncertainties of itself, but also the uncertainties in the actions of other subsystems and the couplings among subsystems. The available results are restricted only to the case of a single uncertainty source (stochastic disturbances or parameter uncertainty). For the case of stochastic disturbances, a distributed robust MPC (DRMPC) algorithm proposed in [8] can guarantee recursive feasibility and stability of the overall system through the use of the constraint-tightening MPC in [9]. It required that all subsystems must be optimized during each sampling period with communication immediately after each optimization, resulting in excessive communication. Following the work of [8], [10] assumed no instantaneous inter-subsystem exchanges of data and yielded a significant reduction in communication cost by permitting only one subsystem to optimize at each time step. A later cooperative DSMPC method for the case that the states are not measurable was given in [11], where the probabilistic constraints were converted into a set of deterministic constraints by making explicit use of the distributions of disturbances, measurement noise and state estimation errors. This strategy was both recursively feasible and computationally tractable, and meanwhile, leads to a more coordinated response and better global performance. For the case of parameter uncertainty, a DSMPC algorithm based on generalized polynomial chaos expansions (GPCEs) was developed in [12], which imposed no restrictions on the distributions of the uncertain parameters. However, if one aims at achieving approximation with high precision, this approach might involve too many constraints, thereby resulting in an overhead cost in computation time.

Motivated by above, using the tube invariant set theory [13, 14], this paper considers a more challenging problem of DSMPC for a group of linear subsystems in simultaneous presence of parameter uncertainty and stochastic disturbances as well as coupled probabilistic constraints. To make the optimization problem tractable in a distributed manner, we adopt the distributed update strategy of [10] that only one subsystem is permitted to optimize at each time step. To the best of our knowledge, it is the first time that a relatively complete theory of DSMPC, including probabilistic constraint handling, recursive feasibility and stability, is established in the context of both model uncertainty and exogenous disturbances. The main attributes of the proposed algorithm are summarized below. 
(i) Inspired by $[6,13,15]$, the uncertain part of the state trajectory is decomposed into two parts: one is handled by calculating a sufficient tightening margin offline, whereas the other is handled by using polytopic tubes with facets of fixed orientation and online adjustable scalings. Both local and coupled probabilistic constraints are then reformulated as linear deterministic constraints, further leading to a convex optimization problem, which can be efficiently solved.

(ii) To ensure coupled probabilistic constraint satisfaction under the distributed update strategy in [10], the constraints derived in the centralized implementation are modified and the resulting constraints involve only the decision variables of a local subsystem and the prior information collected from coupled subsystems.

(iii) Through designing terminal sets as a robust positively invariant set and an invariant hypercube with a parameter chosen from a given interval, the DSMPC algorithm is proven to be recursively feasible and the resulting closed-loop system is stable.

(iv) Exchange of data among subsystems is only required after one subsystem optimization at each time step. Less communication cost and greater flexibility in communications are offered than other DMPC strategies $[8,16,17,18]$, wherein all subsystems are required to optimize and communicate during a sampling period.

The remainder of this paper is organized as follows. The problem statement is presented in Section 2. In Section 3, probabilistic constraints are reformulated into tractable ones. In Section 4, a centralized SMPC problem is derived, starting with a suitable terminal set construction. The main results of the paper are developed in Section 5, where a DSMPC algorithm and its properties are established. In Section 6, the implementation and the distributed nature of the algorithm are discussed. A numerical example is provided in Section 7 and conclusions are drawn in Section 8.

Notation 1.1

Let $\mathbb{N} \triangleq\{0,1, \ldots\}$. For some $q, s \in \mathbb{N}$ and $q<s$, let $\mathbb{N}_{<q}, \mathbb{N}_{\leq q}, \mathbb{N}_{>q}, \mathbb{N}_{\geq q}$, and $\mathbb{N}_{[q, s]}$ denote the sets $\{r \in \mathbb{N} \mid r<q\},\{r \in \mathbb{N} \mid r \leq q\},\{r \in \mathbb{N} \mid r>q\},\{r \in \mathbb{N} \mid r \geq q\}$, and $\{r \in \mathbb{N} \mid q \leq r \leq s\}$, respectively. For any vectors $x_{i}, i \in \mathbb{N}$, let $\sum_{i=s}^{q} x_{i}=\mathbf{0}$ if $q, s \in \mathbb{N}$ and $q<s$. The inequality sign, absolute value $|\cdot|$ and max operations apply elementwise to vectors. $\|\cdot\|_{\infty}$ denotes the vector infinity norm or the corresponding induced matrix norm. $\|x\|_{W}^{2}$ denotes $x^{T} W x$. For sets $\mathcal{A}$ and $\mathcal{B}$, the Cartesian product $\mathcal{A} \times \mathcal{B}$ is the set of all ordered pairs $(a, b)$, where $a \in \mathcal{A}$ and $b \in \mathcal{B}$. A nonnegative matrix $A \geq 0$ is a matrix in which each element is nonnegative, $\mathbf{0}$ is the zero vector or zero matrix whose elements are all zero, and $\mathbf{1}$ is the vector whose elements are all one. Let $\mathrm{Pr}$ denote the probability and let $\mathcal{B}_{\gamma}^{+} \triangleq\left\{\alpha \mid\|\alpha\|_{\infty} \leq \gamma, \alpha \geq \mathbf{0}\right\}$.

For the sake of readability, all the proofs are moved to the Appendix.

\section{PROBLEM STATEMENT}

Consider a system of $N_{p}$ discrete-time dynamically decoupled stochastic linear subsystems

$$
\begin{gathered}
x_{p}(k+1)=A_{p}(k) x_{p}(k)+B_{p}(k) u_{p}(k)+w_{p}(k), \\
\forall k \in \mathbb{N}, p \in \mathcal{P} \triangleq\left\{1,2, \ldots, N_{p}\right\},
\end{gathered}
$$

where $x_{p} \in \mathbb{R}^{N_{x, p}}, u_{p} \in \mathbb{R}^{N_{u, p}}$, and $w_{p} \in \mathbb{R}^{N_{w, p}}$ denote the state, the control input, and the disturbance of subsystem $p$, respectively. Assume that subsystem $p$ is stabilizable for the existence of a stabilizing feedback control. The disturbance sequence $\left\{w_{p}(k), k \in \mathbb{N}\right\}$ is i.i.d. and the distributions of the elements are continuous and compactly supported such that $w_{p}(k) \in W_{p} \triangleq$ $\left\{w_{p}|| w_{p} \mid \leq \alpha_{p}\right\}$, where $\alpha_{p}=\left[\begin{array}{llll}\alpha_{p, 1} & \alpha_{p, 2} & \cdots & \alpha_{p, N_{w, p}}\end{array}\right]^{T}>\mathbf{0}$. The uncertain subsystem matrices $A_{p}(k)$ and $B_{p}(k)$ are

$$
\left[A_{p}(k) B_{p}(k)\right]=\left[A_{p}^{0} B_{p}^{0}\right]+\sum_{j=1}^{L}\left[A_{p}^{(j)} B_{p}^{(j)}\right] q_{p}^{(j)}(k)
$$


where $q_{p}^{(j)}(k) \geq 0, j \in \mathcal{J} \triangleq\{1,2, \ldots, L\}$, are random variables such that $\sum_{j=1}^{L} q_{p}^{(j)}(k)=1$. For a fixed vertex $j$, the sequence $\left\{q_{p}^{(j)}(k), k \in \mathbb{N}\right\}$ is temporally i.i.d..

Define the control input sequence predicted at time $k$ as

$$
\begin{aligned}
& u_{p}(i \mid k)=K_{p} x_{p}(i \mid k)+c_{p}(i \mid k), i \in \mathbb{N}, \\
& c_{p}(i \mid k)=\mathbf{0}, i \in \mathbb{N}_{\geq N},
\end{aligned}
$$

where given a finite prediction horizon $N, c_{p}(i \mid k)$ are optimization variables for $i \in \mathbb{N}_{\leq N-1}$ and define $\mathbf{c}_{p}(k) \triangleq\left[c_{p}^{T}(k) c_{p}^{T}(1 \mid k) \cdots c_{p}^{T}(N-1 \mid k)\right]^{T}$. State feedback matrix $K_{p}$ is determined offline to stabilize subsystem (1) and may, for instance, be chosen to be optimal in the nominal LQ sense or in a worst case sense (for more details, see Chapter 4 of [19]). One strength of the pre-stabilizing action of $u_{p}=K_{p} x_{p}$ is that, under the assumption that $A_{p}(k)+B_{p}(k) K_{p}$ is more contractive than $A_{p}(k)$, it will reduce the effect of uncertainty on the predictions and thereby restrict the growth of tube cross-section.

Our objective is to regulate each subsystem's state to a neighborhood of the origin, while also to guarantee the satisfaction of both local and coupled probabilistic constraints,

$$
\begin{aligned}
& \operatorname{Pr}\left\{\eta_{p}^{T} x_{p}(k) \leq h_{p}\right\} \geq l_{p}, \forall p \in \mathcal{P}, \\
& \operatorname{Pr}\left\{\sum_{p=1}^{N_{p}} \mu_{c p}^{T} x_{p}(k) \leq b_{c}\right\} \geq \tilde{l}_{c}, \forall c \in \mathcal{C} \triangleq\left\{1,2, \ldots, N_{c}\right\},
\end{aligned}
$$

where $\eta_{p} \in \mathbb{R}^{N_{x, p}}$ and $\mu_{c p} \in \mathbb{R}^{N_{x, p}} ; h_{p}$ and $b_{c}$ are fixed scalars; $l_{p} \in[0,1]$ and $\tilde{l}_{c} \in[0,1]$ are prescribed thresholds. Note that hard constraints can be treated as a special case of the probabilistic constraints (3)-(4) by setting $l_{p}=\tilde{l}_{c}=1$.

To facilitate the problem formulation, as in [10], denote by $\mathcal{P}_{c}$ the set of all subsystems involved in coupled constraint $c$,

$$
\mathcal{P}_{c} \triangleq\left\{p \in \mathcal{P} \mid \mu_{c p} \neq \mathbf{0}\right\},
$$

and denote by $\mathcal{C}_{p}$ the set of all coupled constraints involving subsystem $p$,

$$
\mathcal{C}_{p} \triangleq\left\{c \in \mathcal{C} \mid \mu_{c p} \neq \mathbf{0}\right\}
$$

Then we let $\mathcal{Q}_{p}$ denote the set of all other subsystems coupled to subsystem $p$,

$$
\mathcal{Q}_{p} \triangleq\left(\bigcup_{c \in \mathcal{C}_{p}} \mathcal{P}_{c}\right) \backslash\{p\}
$$

\section{PROBABILISTIC CONSTRAINT HANDLING STRATEGY}

In this paper, a DSMPC algorithm is presented to address the problem stated in Section 2. The first step in the study of DSMPC is to handle probabilistic constraints. For this, an effective strategy is developed in this section to transform the probabilistic constraints into deterministic ones by employing tube approaches, in which the constraints are tighten on the nominal model by taking the effect of the uncertainties into account.

For subsystem $p$, the state trajectory predicted at time $k$ is decomposed into a nominal component $z_{p}(i \mid k)$ and an uncertain component $e_{p}(i \mid k)$, which evolve as

$$
\begin{aligned}
x_{p}(i \mid k)= & z_{p}(i \mid k)+e_{p}(i \mid k), \\
z_{p}(i+1 \mid k)= & \Psi_{p}^{0} z_{p}(i \mid k)+B_{p}^{0} c_{p}(i \mid k), \\
e_{p}(i+1 \mid k)= & \Psi_{p}(i \mid k) e_{p}(i \mid k)+\bar{\Psi}_{p}(i \mid k) z_{p}(i \mid k) \\
& +\bar{B}_{p}(i \mid k) c_{p}(i \mid k)+w_{p}(i \mid k),
\end{aligned}
$$


where $\quad \Psi_{p}^{0} \triangleq A_{p}^{0}+B_{p}^{0} K_{p}, \quad \Psi_{p}(i \mid k) \triangleq A_{p}(i \mid k)+B_{p}(i \mid k) K_{p}, \quad \bar{\Psi}_{p}(i \mid k) \triangleq \Psi_{p}(i \mid k)-\Psi_{p}^{0}, \quad$ and $\bar{B}_{p}(i \mid k) \triangleq B_{p}(i \mid k)-B_{p}^{0}$. According to (5), probabilistic constraints (3)-(4) can be expressed with respect to the predicted state sequences for $i \in \mathbb{N}_{\geq 1}$ as

$$
\begin{aligned}
& \operatorname{Pr}\left\{\eta_{p}^{T} z_{p}(i \mid k)+\eta_{p}^{T} e_{p}(i \mid k) \leq h_{p}\right\} \geq l_{p}, \\
& \operatorname{Pr}\left\{\sum_{p=1}^{N_{p}}\left[\mu_{c p}^{T} z_{p}(i \mid k)+\mu_{c p}^{T} e_{p}(i \mid k)\right] \leq b_{c}\right\} \geq \tilde{l}_{c} .
\end{aligned}
$$

From (7), $e_{p}(i \mid k)$ involves both the uncertainties and the decision variables. Therefore, it is intractable to calculate the probabilistic distribution of $e_{p}(i \mid k)$ given the distribution of $w_{p}$. To remedy this, following [6], $e_{p}(i \mid k)$ is further split into two parts, which are given for $i \in \mathbb{N}$ as

$$
\begin{aligned}
e_{p}(i \mid k)= & \varepsilon_{p}(i \mid k)+\zeta_{p}(i \mid k), \\
\varepsilon_{p}(i+1 \mid k)= & \Psi_{p}^{0} \varepsilon_{p}(i \mid k)+w_{p}(i \mid k) \\
\zeta_{p}(i+1 \mid k)= & \Psi_{p}(i \mid k) \zeta_{p}(i \mid k)+\bar{\Psi}_{p}(i \mid k) z_{p}(i \mid k) \\
& +\bar{B}_{p}(i \mid k) c_{p}(i \mid k)+\bar{\Psi}_{p}(i \mid k) \varepsilon_{p}(i \mid k),
\end{aligned}
$$

with the initial conditions $\varepsilon_{p}(k)=\mathbf{0}$ and $z_{p}(k)+\zeta_{p}(k)=x_{p}(k)$. Below, according to the different probabilistic nature, the two components of $e_{p}$ (i.e. $\varepsilon_{p}$ and $\zeta_{p}$ ) in (8)-(9) will be treated separately based on ideas from Tube MPC $[13,15]$.

\subsection{Handling $\varepsilon_{p}$ using the distribution of $w_{p}$}

Note that the evolution of $\varepsilon_{p}$ in (11) is described by a linear time-invariant system subject to disturbances, which is similar to the uncertain systems considered in [15]. Following the result in [15], by making use of probabilistic distributions of $w_{p}$ explicitly, the tightened constraints on the nominal predictions and the other random variable $\zeta_{p}$ are derived in Theorem 3.1, which ensure the satisfactions of (8)-(9) and carry the necessary guarantee of recursive feasibility. The evolution of $\zeta_{p}$ will be discussed in the next section.

\section{Theorem 3.1}

(Conditions for Constraint Satisfaction). The satisfactions of probabilistic constraints (8)-(9) are ensured, if $\mathbf{c}_{p}(k)$ and $z_{p}(0 \mid k)$ for each $p \in \mathcal{P}$ satisfy the recursively feasible constraints, for $i \in \mathbb{N}_{\geq 1}$,

$$
\begin{aligned}
& \eta_{p}^{T} H_{p}^{i} \mathbf{c}_{p}(k)+\eta_{p}^{T}\left(\Psi_{p}^{0}\right)^{i} z_{p}(0 \mid k)+\eta_{p}^{T} \zeta_{p}(i \mid k) \leq h_{p}-\beta_{p}^{i}, \forall p \in \mathcal{P}, \\
& \sum_{q \in \mathcal{P}_{c}}\left[\mu_{c q}^{T} H_{q}^{i} \mathbf{c}_{q}(k)+\mu_{c q}^{T}\left(\Psi_{q}^{0}\right)^{i} z_{q}(0 \mid k)+\mu_{c q}^{T} \zeta_{q}(i \mid k)\right] \leq b_{c}-\kappa_{c}^{i}, \forall c \in \mathcal{C},
\end{aligned}
$$

where $H_{p}^{i} \triangleq\left[\begin{array}{lllllll}\left(\Psi_{p}^{0}\right)^{i-1} B_{p}^{0} & \left(\Psi_{p}^{0}\right)^{i-2} B_{p}^{0} & \cdots & B_{p}^{0} & \mathbf{0} & \cdots & \mathbf{0}\end{array}\right]$. The constraint tightening parameters $\beta_{p}^{i}$ and $\kappa_{c}^{i}$ are given by, for $i \in \mathbb{N}_{\geq 1}$,

$$
\beta_{p}^{i} \triangleq \gamma_{p}+\sum_{j=1}^{i-1} a_{p}^{j}, \kappa_{c}^{i} \triangleq \xi_{c}+\sum_{j=1}^{i-1} d_{c}^{j},
$$

where

$$
a_{p}^{j} \triangleq \max _{w_{p} \in W_{p}} \eta_{p}^{T}\left(\Psi_{p}^{0}\right)^{j} w_{p}=\left|\eta_{p}^{T}\left(\Psi_{p}^{0}\right)^{j}\right| \alpha_{p}, d_{c}^{j} \triangleq \max _{w_{q} \in W_{q}} \sum_{q \in \mathcal{P}_{c}} \mu_{c q}^{T}\left(\Psi_{q}^{0}\right)^{j} w_{q}=\sum_{q \in \mathcal{P}_{c}}\left|\mu_{c q}^{T}\left(\Psi_{q}^{0}\right)^{j}\right| \alpha_{q},
$$

$\gamma_{p}$ and $\xi_{c}$ are the minimum values such that

$$
\operatorname{Pr}\left\{\eta_{p}^{T} w_{p}(k) \leq \gamma_{p}\right\}=l_{p}, \operatorname{Pr}\left\{\sum_{q \in \mathcal{P}_{c}} \mu_{c q}^{T} w_{q}(k) \leq \xi_{c}\right\}=\tilde{l}_{c} .
$$


The interested reader may refer to [15] for a similar proof.

Remark 3.1

(Properties of $\beta_{p}^{i}$ and $\kappa_{c}^{i}$ ). The sequences $\left\{\beta_{p}^{i}, i \in \mathbb{N}_{\geq 1}\right\}$ and $\left\{\kappa_{c}^{i}, i \in \mathbb{N}_{\geq 1}\right\}$ in (13)-(14) are monotonically non-decreasing and converge to limits $\bar{\beta}_{p}$ and $\bar{\kappa}_{c}$ respectively, which will be used later (see Section 4.1) to design terminal sets. Furthermore, the arbitrarily tight upper bounds on $\bar{\beta}_{p}$ and $\bar{\kappa}_{c}$ can be computed offline (see Corollary 4 in [15]).

\subsection{Handling $\zeta_{p}$ using polytopic tubes}

Different from $\varepsilon_{p}$, it is impossible to compute offline the probabilistic distribution of $\zeta_{p}$. The reason is that the dynamics of $\zeta_{p}$ in (12) involves both the nominal states and the decision variables. To handle the uncertain part $\zeta_{p}$ in (13)-(14), a sequence of sets (i.e. a tube) with bounding facets of fixed orientation is constructed online to characterize the evolution of $\zeta_{p}$ instead of using probabilistic distributions [13].

Suppose that the tube has polytopic cross-sections and is parameterized by

$$
\Pi_{p}(i \mid k) \triangleq\left\{\zeta_{p}(i \mid k) \mid V_{p} \zeta_{p}(i \mid k) \leq \theta_{p}(i \mid k)\right\}, i \in \mathbb{N},
$$

where $V_{p} \in \mathbb{R}^{N_{v, p} \times N_{x, p}}$ is chosen offline to determine the directions of facets of the tube and $\theta_{p} \in \mathbb{R}^{N_{v, p}}$ is optimized online to determine the distances from the origin to the facets.

In order to ensure that initially bounding $\zeta_{p}(0 \mid k)$ in a given tube cross-section $\Pi_{p}(0 \mid k)$ implies that $\zeta_{p}(i \mid k)$ will stay in $\Pi_{p}(i \mid k)$ at all subsequent prediction times, the choice of $\theta_{p}(i \mid k)$ is required to satisfy

$$
\left\{\zeta_{p}(i+1 \mid k) \mid \zeta_{p}(i \mid k) \in \Pi_{p}(i \mid k)\right\} \subseteq \Pi_{p}(i+1 \mid k) .
$$

In addition, the choice of $\theta_{p}(i \mid k)$ must also ensure the satisfactions of (13)-(14), given that $\zeta_{p}(i \mid k)$ is contained in $\Pi_{p}(i \mid k)$ for each subsystem, i.e.,

$$
\begin{gathered}
\Pi_{p}(i \mid k) \subseteq\left\{\zeta_{p}(i \mid k) \mid(13) \text { holds }\right\} \\
\left\{\left(\zeta_{1}(i \mid k), \ldots, \zeta_{N_{p}}(i \mid k)\right) \mid \zeta_{p}(i \mid k) \in \Pi_{p}(i \mid k), \forall p \in \mathcal{P}\right\} \subseteq\left\{\left(\zeta_{1}(i \mid k), \ldots, \zeta_{N_{p}}(i \mid k)\right) \mid(14) \text { holds }\right\}
\end{gathered}
$$

The following lemma can be used to express the inclusion conditions (16)-(18) as the equivalent linear constraints.

Lemma 3.1

[20] (Necessary and Sufficient Conditions for Set Inclusions). The inclusion $\left\{x \mid F_{1} x \leq b_{1}\right\} \subseteq\{x \mid$ $\left.F_{2} x \leq b_{2}\right\}$ holds if and only if there exists a non-negative matrix $H$ such that

$$
H F_{1}=F_{2}, H b_{1} \leq b_{2} .
$$

By using Lemma 3.1, Theorem 3.2 provides necessary and sufficient conditions to ensure constraint (16) satisfaction.

\section{Theorem 3.2}

(Conditions for Tube Cross-section Evolution). For any $\zeta_{p}(i \mid k) \in \Pi_{p}(i \mid k)$, a necessary and sufficient condition for $\zeta_{p}(i+1 \mid k) \in \Pi_{p}(i+1 \mid k)$ is that for $i \in \mathbb{N}$, there exist nonnegative matrices $M_{p}^{(j)} \geq$ $0, j \in \mathcal{J}$, such that

$$
\begin{gathered}
M_{p}^{(j)} V_{p}=V_{p}\left(\Psi_{p}^{0}+\Psi_{p}^{(j)}\right), \\
M_{p}^{(j)} \theta_{p}(i \mid k)+V_{p} \Psi_{p}^{(j)} z_{p}(i \mid k)+V_{p} B_{p}^{(j)} c_{p}(i \mid k)+d_{p}^{(j)}(i) \leq \theta_{p}(i+1 \mid k),
\end{gathered}
$$

where $d_{p}^{(j)}(i) \triangleq \max _{w_{p} \in W_{p}} V_{p} \Psi_{p}^{(j)} \varepsilon_{p}(i \mid k)$. 


\section{Remark 3.2}

(Properties of $d_{p}^{(j)}(i)$ ). According to the definition of $\varepsilon_{p}$ in (11), the value of $d_{p}^{(j)}(i)$ does not depend on the system state and can be calculated offline by solving a finite number of linear programming problems. Furthermore, as $\bar{\beta}_{p}$ and $\bar{\kappa}_{c}$ in Remark 3.1, for each vertex $j \in \mathcal{J}$, the sequence $\left\{d_{p}^{(j)}(i), i \in \mathbb{N}\right\}$ converges to a limit $\bar{d}_{p}^{(j)}$, on which an arbitrarily close upper bound can be obtained.

Similar treatments can be used to obtain necessary and sufficient conditions for the satisfaction of (17)-(18). The result is given below directly without proof.

\section{Theorem 3.3}

(Conditions for Constraint Satisfaction in Tubes). Provided that $\zeta_{p}(i \mid k) \in \Pi_{p}(i \mid k)$ for each $p \in \mathcal{P}$, the constraints (13)-(14) for $i \in \mathbb{N}_{\geq 1}$ are satisfied if and only if there exist nonnegative matrices $M_{p} \geq 0$ and $M_{c p} \geq 0$ satisfying

$$
\begin{gathered}
M_{p} V_{p}=\eta_{p}^{T}, \\
M_{p} \theta_{p}(i \mid k)+\beta_{p}^{i}+\eta_{p}^{T} H_{p}^{i} \mathbf{c}_{p}(k)+\eta_{p}^{T}\left(\Psi_{p}^{0}\right)^{i} z_{p}(0 \mid k) \leq h_{p},
\end{gathered}
$$

and

$$
\begin{gathered}
M_{c p} V_{p}=\mu_{c p}^{T} \\
\sum_{q \in \mathcal{P}_{c}}\left[\mu_{c q}^{T} H_{q}^{i} \mathbf{c}_{q}(k)+\mu_{c q}^{T}\left(\Psi_{q}^{0}\right)^{i} z_{q}(0 \mid k)+M_{c q} \theta_{q}(i \mid k)\right]+\kappa_{c}^{i} \leq b_{c} .
\end{gathered}
$$

Treating $M_{p}^{(j)}, M_{p}, M_{c p}$, and $V_{p}$ as decision variables will result in nonlinear constraints (20)(25) and further intractable online optimization problem. Instead, we select $M_{p}^{(j)}, M_{p}, M_{c p}$, and $V_{p}$ offline to guarantee the satisfactions of (20), (22), and (24), and only leave (21), (23), and (25) as the online constraints, which will imply a tractable convex optimization problem (see Section 6.3). The suitable choices and practical considerations for $M_{p}^{(j)}, M_{p}, M_{c p}$, and $V_{p}$ will be discussed in Section 6.1.

\section{CENTRALIZED SMPC ALGORITHM}

In this section, a centralized SMPC controller is developed to achieve the control objective. We first focus the investigations on the construction of suitable terminal constraints, which make the decision variables finite dimensional in an infinite horizon optimization problem. Subsequently, by combining these terminal constraints and the linear constraints (21), (23), and (25), a centralized infinite-horizon SMPC optimization problem is formulated. The extension to a distributed implementation will be discussed in Section 5.

\subsection{Construction of Terminal Sets}

To account for constraints after the $N_{1}$ th prediction time with $N_{1} \in \mathbb{N}_{>N}$, we design terminal sets for $z_{p}\left(N_{1} \mid k\right)$ and $\theta_{p}\left(N_{1} \mid k\right)$. Similar to classical MPC schemes, the terminal sets should be positively invariant and moreover, inside them, constraints (21), (23), and (25) should be satisfied. For $i \in \mathbb{N}_{\geq N}$, with $c_{p}(i \mid k)=\mathbf{0}$, (6) and (21) can be rewritten as

$$
\begin{gathered}
z_{p}(i+1 \mid k)=\Psi_{p}^{0} z_{p}(i \mid k), \\
M_{p}^{(j)} \theta_{p}(i \mid k)+V_{p} \Psi_{p}^{(j)} z_{p}(i \mid k)+d_{p}^{(j)}(i) \leq \theta_{p}(i+1 \mid k) .
\end{gathered}
$$

Note that the dynamic of $z$ in (26) is not affected by $\theta_{p}$. Thus, first we design a terminal set for $z$ and second a terminal set for $\theta_{p}$. Require $z_{p}(N \mid k)$ to lie within a set $\chi_{p}^{0}$, which is robust positively invariant for the system $z_{p}(k+1)=\Psi_{p}^{0} z_{p}(k)+\left(\Psi_{p}^{0}\right)^{N} w_{p}(k)$. Hence, at prediction time $k+N_{1}$, $z_{p}\left(N_{1} \mid k\right)$ must enter a smaller set defined by $\chi_{p}^{l} \triangleq\left(\Psi_{p}^{0}\right)^{l} \chi_{p}^{0}, l=N_{1}-N$. It is easy to see that $\chi_{p}^{l}$ is 
positively invariant with respect to (26) and can therefore be chosen as the terminal set for $z_{p}\left(N_{1} \mid k\right)$. Based on $\chi_{p}^{l}$, an invariant hypercube is then designed in Theorem 4.1 to serve as a terminal set for $\theta_{p}\left(N_{1} \mid k\right)$.

Theorem 4.1

(Terminal Constraints). Define $v_{p, l}^{(j)} \triangleq \max _{z_{p} \in \chi_{p}^{l}} V_{p} \Psi_{p}^{(j)} z_{p}, f_{p, l} \triangleq \max _{z_{p} \in \chi_{p}^{l}} \eta_{p}^{T} z_{p}$, and $f_{c p, l} \triangleq$ $\max _{z_{p} \in \chi_{p}^{l}} \mu_{c p}^{T} z_{p}$. If $\tilde{\theta}_{p}$ satisfies the following conditions

$$
\begin{gathered}
\tilde{\theta}_{p} \geq \frac{\max _{j}\left(\left\|v_{p, l}^{(j)}\right\|_{\infty}+\left\|\bar{d}_{p}^{(j)}\right\|_{\infty}\right)}{1-\max _{j}\left\|M_{p}^{(j)}\right\|_{\infty}}, \\
\tilde{\theta}_{p} \leq \frac{h_{p}-\bar{\beta}_{p}-\left\|f_{p, l}\right\|_{\infty}}{\left\|M_{p}\right\|_{\infty}}, \\
\sum_{p=1}^{N_{p}}\left\|M_{c p}\right\|_{\infty} \tilde{\theta}_{p} \leq b_{c}-\bar{\kappa}_{c}-\sum_{p=1}^{N_{p}}\left\|f_{c p, l}\right\|_{\infty},
\end{gathered}
$$

then the set $\chi_{p}^{l} \times \mathcal{B}_{\tilde{\theta}_{p}}^{+}$is invariant for the following system,

$$
\begin{aligned}
& z_{p}(i+1 \mid k)=\Psi_{p}^{0} z_{p}(i \mid k), \\
& \theta_{p}(i+1 \mid k)=\max _{j}\left\{M_{p}^{(j)} \theta_{p}(i \mid k)+d_{p}^{(j)}(i)+V_{p} \Psi_{p}^{(j)} z_{p}(i \mid k)\right\}
\end{aligned}
$$

and the satisfactions of (21), (23), and (25) for $i \in \mathbb{N}_{\geq N_{1}}$ can be ensured if $\left(z_{p}\left(N_{1} \mid k\right), \theta_{p}\left(N_{1} \mid k\right)\right) \in$ $\chi_{p}^{l} \times \mathcal{B}_{\tilde{\theta}_{p}}^{+}$.

From the fact that $\lim _{l \rightarrow \infty} v_{p, l}^{(j)}=0, \lim _{l \rightarrow \infty} f_{p, l}=0$, and $\lim _{l \rightarrow \infty} f_{c p, l}=0$, the existence of these terminal sets, i.e., the existence of $\tilde{\theta}_{p}$ for each subsystem satisfying (28)-(30), can be ensured if $l=N_{1}-N$ is chosen to be sufficiently large and the following conditions are satisfied [14]:

$$
\begin{gathered}
\frac{\left\|M_{p}\right\|_{\infty} \max _{j}\left\|\bar{d}_{p}^{(j)}\right\|_{\infty}}{1-\max _{j}\left\|M_{p}^{(j)}\right\|_{\infty}}<h_{p}-\bar{\beta}_{p}, \forall p \in \mathcal{P}, \\
\sum_{p=1}^{N_{p}} \frac{\left\|M_{c p}\right\|_{\infty} \max _{j}\left\|\bar{d}_{p}^{(j)}\right\|_{\infty}}{1-\max _{j}\left\|M_{p}^{(j)}\right\|_{\infty}}<b_{c}-\bar{\kappa}_{c}, \forall c \in \mathcal{C} .
\end{gathered}
$$

In order to satisfy the coupling condition (30), the terminal sets of all subsystems are required to be designed centrally. However, note that the centralized design of terminal sets is implemented offline.

\subsection{Optimization Problem Formulation}

With the constraints (21), (23), (25), and the terminal constraints given in Theorem 4.1, we are now ready to define the optimization problem used in the centralized SMPC scheme. In particular, for any $k \in \mathbb{N}$ and any state $x_{1}(k), \ldots, x_{N_{p}}(k)$, the centralized optimal control problem $\mathbf{P}^{C}\left(x_{1}(k), \ldots, x_{N_{p}}(k)\right)$ is defined as follows:

$$
\min _{\substack{\theta_{p}(0 \mid k), \ldots, \theta_{p}\left(N_{1} \mid k\right), \mathbf{c}_{p}(k), z_{p}(0 \mid k), p \in \mathcal{P}}} J(k)=\sum_{p=1}^{N_{p}} \sum_{i=0}^{\infty}\left[\left\|z_{p}(i \mid k)\right\|_{Q_{p}}^{2}+\left\|K_{p} z_{p}(i \mid k)+c_{p}(i \mid k)\right\|_{R_{p}}^{2}\right]
$$

subject to $\forall p \in \mathcal{P}$ :

$$
\begin{gathered}
V_{p}\left(x_{p}(k)-z_{p}(0 \mid k)\right) \leq \theta_{p}(0 \mid k) \\
\forall i \in \mathbb{N}_{\leq N_{1}-1}: z_{p}(i+1 \mid k)=\Psi_{p}^{0} z_{p}(i \mid k)+B_{p}^{0} c_{p}(i \mid k)
\end{gathered}
$$




$$
\begin{aligned}
\forall i \in \mathbb{N}_{\leq N_{1}-1}, \forall j \in \mathcal{J}: & M_{p}^{(j)} \theta_{p}(i \mid k)+V_{p} \Psi_{p}^{(j)} z_{p}(i \mid k)+V_{p} B_{p}^{(j)} c_{p}(i \mid k)+d_{p}^{(j)}(i) \\
& \leq \theta_{p}(i+1 \mid k) \\
\forall i \in \mathbb{N}_{\left[1, N_{1}-1\right]}: & M_{p} \theta_{p}(i \mid k)+\beta_{p}^{i}+\eta_{p}^{T} H_{p}^{i} \mathbf{c}_{p}(k)+\eta_{p}^{T}\left(\Psi_{p}^{0}\right)^{i} z_{p}(0 \mid k) \leq h_{p} \\
\forall i \in \mathbb{N}_{\left[1, N_{1}-1\right]}, \forall c \in \mathcal{C}: & \sum_{q \in \mathcal{P}_{c}}\left[\mu_{c q}^{T} H_{q}^{i} \mathbf{c}_{q}(k)+\mu_{c q}^{T}\left(\Psi_{q}^{0}\right)^{i} z_{q}(0 \mid k)+M_{c q} \theta_{q}(i \mid k)\right]+\kappa_{c}^{i} \leq b_{c} \\
& z_{p}(N \mid k) \in \chi_{p}^{0} \\
& \left\|\theta_{p}\left(N_{1} \mid k\right)\right\|_{\infty} \leq \tilde{\theta}_{p}
\end{aligned}
$$

where $Q_{p}$ and $R_{p}$ are symmetric positive-definite matrices. In $\mathbf{P}^{C}\left(x_{1}(k), \ldots, x_{N_{p}}(k)\right),(36)$ and (38) are constraints on the initial tube cross-section and tube cross-section evolution respectively; (37) is the nominal system model; (39) and (40) are used to invoke the local and coupled constraints (3) and (4) respectively; (41) and (42) are terminal constraints. At each time step $k$, all subsystems must sample and send their current states to the centralized control subsystem. After solving $\mathbf{P}^{C}\left(x_{1}(k), \ldots, x_{N_{p}}(k)\right)$, the optimal solution $\theta_{p}^{*}(0 \mid k), \ldots, \theta_{p}^{*}\left(N_{1} \mid k\right), \mathbf{c}_{p}^{*}(k), z_{p}^{*}(0 \mid k)$ is communicated to each subsystem $p$. Subsequently, all subsystems apply $u_{p}(k)=K_{p} x_{p}(k)+c_{p}^{*}(k)$ to control. Provided that the centralized control subsystem is one of the subsystems $p \in \mathcal{P}$, centralized SMPC algorithm requires $2\left(N_{p}-1\right)$ data exchanges per optimization, which must be completed during one sampling period.

\section{DSMPC ALGORITHM}

For large-scale systems, it is difficult to control with centralized MPC due to the required inherent computational complexity, communication bandwidth limitations and the reliance on a single processor. Thus DMPC is usually adopted, despite the fact that it generally degrades the performance compared with centralized MPC scheme. This section aims to extend the centralized SMPC in Section 4 to a distributed form. A distributed update strategy adopted is first given as follows. At each time $k$, only one subsystem, defined as $p_{k}$, is permitted to optimize for a new solution $\mathbf{c}_{p_{k}}(k)$. Meanwhile, all other subsystems $p \neq p_{k}$ adopt the feasible solutions

$$
\mathbf{c}_{p}(k)=\left[c_{p}^{T}(1 \mid k-1) \cdots c_{p}^{T}(N-1 \mid k-1) \mathbf{0}\right]^{T},
$$

which are the tails of their previous solutions $\mathbf{c}_{p}(k-1)$ augmented with $\mathbf{0}$. It is noted that we have no specific assumptions on the update sequence $\left\{p_{1}, \ldots, p_{k}, p_{k+1}, \ldots\right\}$, which can be tailored according to the actual situations. The strategy for constructing the update sequence will be explored in Section 6.2.

\subsection{Distributed Implementation Algorithm}

We define the cost function for subsystem $p$ as

$$
J_{p}(k)=\sum_{i=0}^{\infty}\left[\left\|z_{p}(i \mid k)\right\|_{Q_{p}}^{2}+\left\|K_{p} z_{p}(i \mid k)+c_{p}(i \mid k)\right\|_{R_{p}}^{2}\right] .
$$

Lemma 5.1 shows that (44) can be expressed as a quadratic form of the decision variables $z_{p}(0 \mid k)$ and $\mathbf{c}_{p}(k)$.

\section{Lemma 5.1}

(Quadratic Formulation of the Cost). Let $W_{p}$ be a solution of the Lyapunov equation

$$
W_{p}-\left[\begin{array}{cc}
\Psi_{p}^{0} & B_{p}^{0} E_{p} \\
\mathbf{0} & T_{p}
\end{array}\right]^{T} W_{p}\left[\begin{array}{cc}
\Psi_{p}^{0} & B_{p}^{0} E_{p} \\
\mathbf{0} & T_{p}
\end{array}\right]=\bar{Q}_{p}
$$

where $\bar{Q}_{p}=\left[\begin{array}{cc}Q_{p}+K_{p}^{T} R_{p} K_{p} & K_{p}^{T} R_{p} E_{p} \\ E_{p}^{T} R_{p} K_{p} & E_{p}^{T} R_{p} E_{p}\end{array}\right], E_{p}=\left[\begin{array}{cccc}I & \mathbf{0} & \cdots & \mathbf{0}\end{array}\right]$, and $T_{p}$ is a matrix with the identity matrix on its super-diagonal blocks and zeros elsewhere, then the cost function (44) can 
be rewritten as

$$
J_{p}(k)=\left\|\left[\begin{array}{c}
z_{p}(0 \mid k) \\
\mathbf{c}_{p}(k)
\end{array}\right]\right\|_{W_{p}}^{2} .
$$

By considering constraints (36)-(42) for subsystem $p$ only and modifying coupled constraint (40), the local optimization problem for subsystem $p$ defined by $\mathbf{P}_{p}^{D}\left(x_{p}(k) ; Z_{p}^{*}(k)\right)$ is formulated as follows.

subject to

$$
\min _{\substack{\theta_{p}(0 \mid k), \ldots, \theta_{p}\left(N_{1} \mid k\right), \mathbf{c}_{p}(k), z_{p}(0 \mid k)}} J_{p}(k)=\left\|\left[\begin{array}{c}
z_{p}(0 \mid k) \\
\mathbf{c}_{p}(k)
\end{array}\right]\right\|_{W_{p}}^{2}
$$

$$
\begin{aligned}
& V_{p}\left(x_{p}(k)-z_{p}(0 \mid k)\right) \leq \theta_{p}(0 \mid k) \\
\forall i \in \mathbb{N}_{\leq N_{1}-1}: & z_{p}(i+1 \mid k)=\Psi_{p}^{0} z_{p}(i \mid k)+B_{p}^{0} c_{p}(i \mid k) \\
\forall i \in \mathbb{N}_{\leq N_{1}-1}, \forall j \in \mathcal{J}: & M_{p}^{(j)} \theta_{p}(i \mid k)+V_{p} \Psi_{p}^{(j)} z_{p}(i \mid k)+V_{p} B_{p}^{(j)} c_{p}(i \mid k)+d_{p}^{(j)}(i) \\
& \leq \theta_{p}(i+1 \mid k) \\
\forall i \in \mathbb{N}_{\left[1, N_{1}-1\right]}: & M_{p} \theta_{p}(i \mid k)+\beta_{p}^{i}+\eta_{p}^{T} H_{p}^{i} \mathbf{c}_{p}(k)+\eta_{p}^{T}\left(\Psi_{p}^{0}\right)^{i} z_{p}(k) \leq h_{p} \\
\forall i \in \mathbb{N}_{\left[1, N_{1}-1\right]}, \forall c \in \mathcal{C}_{p}: & M_{c p} \theta_{p}(i \mid k)+\mu_{c p}^{T} H_{p}^{i} \mathbf{c}_{p}(k)+\mu_{c p}^{T}\left(\Psi_{p}^{0}\right)^{i} z_{p}(0 \mid k)+\sum_{q \in \mathcal{P}_{c} \backslash\{p\}}\left[\mu_{c q}^{T} H_{q}^{i} \mathbf{c}_{q}^{*}(k)\right. \\
& \left.+\mu_{c q}^{T}\left(\Psi_{q}^{0}\right)^{i} z_{q}^{*}(0 \mid k)+\Xi_{c q}^{*}(i \mid k)+M_{c q} \theta_{q}^{*}(i \mid k)\right]+\kappa_{c}^{i} \leq b_{c} \\
& z_{p}(N \mid k) \in \chi_{p}^{0} \\
& \left\|\theta_{p}\left(N_{1} \mid k\right)\right\|_{\infty} \leq \tilde{\theta}_{p}
\end{aligned}
$$

Note that in $(40) z_{q}(0 \mid k), \mathbf{c}_{q}(k)$, and $\theta_{q}(i \mid k)$ involving subsystems $q \in \mathcal{Q}_{p}$ are modified to $z_{q}^{*}(0 \mid k)$, $\mathbf{c}_{q}^{*}(k)$, and $\theta_{q}^{*}(i \mid k)$ in (52), which are not affected by the decision variables of subsystem $p$ and can be regarded as fixed values, denoted by $*$. Note also that to ensure the satisfactions of coupled probabilistic constraint in the distributed implementation, new scalar variables $\Xi_{c q}^{*}(i \mid k)$ are introduced in (52). The following part will discuss the detailed method to derive the values of $z_{q}^{*}(0 \mid k), \mathbf{c}_{q}^{*}(k), \theta_{q}^{*}(i \mid k)$, and $\Xi_{c q}^{*}(i \mid k)$ taking into account recursive feasibility of the online optimization problem $\mathbf{P}_{p}^{D}\left(x_{p}(k) ; Z_{p}^{*}(k)\right)$.

Define the last update step of subsystem $q$ as $\hat{k}_{q} \triangleq \max _{k^{\prime} \in\left\{k^{\prime} \in \mathbb{N}_{<k} \mid p_{k^{\prime}}=q\right\}} k^{\prime}$. Then the optimizing subsystem $p$ can construct $\mathbf{c}_{q}^{*}(k), z_{q}^{*}(0 \mid k), \theta_{q}^{*}(i \mid k)$, and $\Xi_{c q}^{*}(i \mid k)$ in (52) by (55)-(59) with the optimal solution $\mathbf{c}_{q}^{*}\left(\hat{k}_{q}\right), z_{q}^{*}\left(0 \mid \hat{k}_{q}\right)$, and $\theta_{q}^{*}\left(i \mid \hat{k}_{q}\right), i \in \mathbb{N}_{\left[2, N_{1}\right]}$, of subsystem $q \in \mathcal{Q}_{p}$ at time $\hat{k}_{q}$.

$$
\begin{aligned}
& \mathbf{c}_{q}^{*}(k)=T_{q}^{k-\hat{k}_{q}} \mathbf{c}_{q}^{*}\left(\hat{k}_{q}\right) \\
& z_{q}^{*}(k)=z_{q}^{*}\left(k-\hat{k}_{q} \mid \hat{k}_{q}\right) \\
& \theta_{q}^{*}(i \mid k)=\theta_{q}^{*}\left(k+i-\hat{k}_{q} \mid \hat{k}_{q}\right), i \in \mathbb{N}_{\left[1, \hat{k}_{q}+N_{1}-k\right]} \\
& \theta_{q}^{*}(i \mid k)=\max _{j}\left\{M_{q}^{(j)} \theta_{q}^{*}\left(k+i-1-\hat{k}_{q} \mid \hat{k}_{q}\right)+V_{q} \Psi_{q}^{(j)} z_{q}^{*}\left(k+i-1-\hat{k}_{q} \mid \hat{k}_{q}\right)+d_{q}^{(j)}\left(N_{1}\right)\right\} \\
& \Xi_{c q}^{*}(i \mid k)=\sum_{l=i}^{k-\hat{k}_{q}-1+i}\left|\mu_{c q}^{T}\left(\Psi_{q}^{0}\right)^{l}\right| \alpha_{q}, i \in \mathbb{N}_{\left[1, N_{1}-1\right]} .
\end{aligned}
$$

Hence, in the optimization problem $\mathbf{P}_{p}^{D}\left(x_{p}(k) ; Z_{p}^{*}(k)\right)$, we use $Z_{p}^{*}(k)$ to denote the set of information collected from all coupled subsystems $q \in \mathcal{Q}_{p}$ at their last update steps, i.e. $Z_{p}^{*}(k) \triangleq$ $\left\{\mathbf{c}_{q}^{*}\left(\hat{k}_{q}\right), z_{q}^{*}\left(0 \mid \hat{k}_{q}\right)\right.$, and $\left.\theta_{q}^{*}\left(i \mid \hat{k}_{q}\right), i \in \mathbb{N}_{\left[2, N_{1}\right]}, q \in \mathcal{Q}_{p}\right\}$, which is required by optimizing subsystem $p$ to evaluate constraint (52).

The DSMPC algorithm can now be summarized below. 


\section{Algorithm 1.}

1) Initialization: Wait for a feasible solution $\left\{\mathbf{c}_{p}(k), z_{p}(0 \mid k), \theta_{p}(0 \mid k), \theta_{p}(1 \mid k), \ldots, \theta_{p}\left(N_{1} \mid k\right)\right\}$ for each $p \in \mathcal{P}$ by solving the centralized problem $\mathbf{P}^{C}\left(x_{1}(k), \ldots, x_{N_{p}}(k)\right)$. If a solution cannot be found, stop.

2) All subsystems $p$ : Apply $u_{p}(k)=K_{p} x_{p}(k)+c_{p}(k)$.

3) All subsystems $p$ : Update $k$ and sample states $x_{p}(k)$.

4) Subsystem $p=p_{k}$ :

a) Solve $\mathbf{P}_{p}^{D}\left(x_{p}(k) ; Z_{p}^{*}(k)\right)$ to obtain $\left\{\mathbf{c}_{p}(k), z_{p}(0 \mid k), \theta_{p}(0 \mid k), \theta_{p}(1 \mid k), \ldots, \theta_{p}\left(N_{1} \mid k\right)\right\}$.

b) Transmit the new solution $\left\{\mathbf{c}_{p}(k), z_{p}(0 \mid k), \theta_{p}(2 \mid k), \ldots, \theta_{p}\left(N_{1} \mid k\right)\right\}$ to subsystems $q \in \mathcal{Q}_{p}$.

c) Set $\hat{k}_{p}=k$.

Subsystems $p \neq p_{k}$ :

a) Construct $\mathbf{c}_{p}(k)=T_{p}^{k-\hat{k}_{p}} \mathbf{c}_{p}\left(\hat{k}_{p}\right)$ directly without solving $\mathbf{P}_{p}^{D}\left(x_{p}(k) ; Z_{p}^{*}(k)\right)$.

5) Go to step 2).

Different from the centralized SMPC, information transmissions in Algorithm 1 are only required after the local optimization problem $\mathbf{P}_{p}^{D}\left(x_{p}(k) ; Z_{p}^{*}(k)\right)$ is solved for a new solution $\left\{\mathbf{c}_{p}(k), z_{p}(0 \mid k), \theta_{p}(0 \mid k), \theta_{p}(1 \mid k), \ldots, \theta_{p}\left(N_{1} \mid k\right)\right\}$. Assuming that the optimizing subsystem is coupled with all other subsystems, Algorithm 1 requires, at most, $\left(N_{p}-1\right)$ data transmissions at each time step, which is less than half of what centralized SMPC requires. In addition, Algorithm 1 achieves greater flexibility in communication. In other alternative DMPC strategies $[8,16,17,18]$, all subsystems optimize and communicate at each time step. A key feature that arises from applying such methods is repeated instantaneous data exchanges between subsystems during a sampling period, either when all subsystems optimize in a sequential way $[8,16]$ or in an iterative way $[17,18]$. As a result, these strategies have poor capacity to tolerate delays in computation and communication. However, Algorithm 1 does not need to communicate immediately after each optimization. The optimizing subsystem allows to transmit the new solution to all coupled subsystems in the whole interval before another subsystem in $\mathcal{Q}_{p}$ optimizes.

\subsection{Main Results}

This section aims at deriving the properties of the proposed DSMPC scheme (Algorithm 1). To have a well-defined control law, we first show that Algorithm 1 can provide the guarantee of recursive feasibility in Theorem 5.1.

Theorem 5.1

(Recursive Feasibility and Constraint Satisfaction). If there exists a feasible (but not necessarily optimal) solution to the optimization problem $\mathbf{P}_{p}^{D}\left(x_{p}\left(k_{0}\right) ; Z_{p}^{*}\left(k_{0}\right)\right)$ for each $p \in \mathcal{P}$ at time $k_{0}$, and the system is controlled according to Algorithm 1, then

(i) all subsequent optimization problems $\mathbf{P}_{p}^{D}\left(x_{p}(k) ; Z_{p}^{*}(k)\right)$ are feasible for $k \in \mathbb{N}_{\geq k_{0}+1}$;

(ii) probabilistic constraints (3) and (4) are satisfied in closed-loop operation for $k \in \mathbb{N}_{\geq k_{0}+1}$.

Based on Theorem 5.1, closed-loop stability under Algorithm 1 is guaranteed in the following theorem.

Theorem 5.2

(Closed-loop Stability). If the optimization problem $\mathbf{P}_{p}^{D}\left(x_{p}\left(k_{0}\right) ; Z_{p}^{*}\left(k_{0}\right)\right)$ is feasible for each $p \in \mathcal{P}$ at time $k_{0}$, then the system is stabilized under Algorithm 1 and the MPC control law converges asymptotically to the unconstrained optimal control law as $k \rightarrow \infty$. 


\section{IMPLEMENTATION AND COMPUTATION}

The purpose of this section is threefold: (i) to put emphasis on the offline implementation about some key parameters (i.e. $\gamma_{p}^{i}, \xi_{c}^{i}, V_{p}, M_{p}^{(j)}, M_{p}$, and $M_{c p}$ ) from a practical perspective; (ii) to discuss the design for update sequence to achieve a flexibility in communication and to reduce the usage of communication resources; (iii) to show the benefit in terms of the structure and computational complexity of the online optimization problem from the use of Algorithm 1.

\subsection{Offline Implementation}

The values of $\gamma_{p}^{i}$ and $\xi_{c}^{i}$ should ideally be calculated by a multivariate convolution, but the computational complexity increases tremendously as the system dimension and the prediction horizon increase. One alternative is to discretize the distributions of the elements of $w_{p}$, and the values of $\gamma_{p}^{i}$ and $\xi_{c}^{i}$ can be approximated using univariate convolutions in polynomial time (see Remark 3.2 in [11]). It is worth noting that $\gamma_{p}^{i}$ and $\xi_{c}^{i}$ are independent of the system states and therefore can be determined offline.

As for $V_{p}$, one way is to select $V_{p}$ such that $\left\{x \mid V_{p} x \leq 1\right\}$ is a $\lambda$-contractive set of the system $x_{p}(k+1)=\Psi_{p}(k) x_{p}(k)$ where $0<\lambda<1$ or, equivalently, an invariant set of the system $x_{p}(k+1)=\frac{\Psi_{p}(k)}{\lambda} x_{p}(k)$ (see [20]). For this choice of $V_{p}$, it follows from the invariance conditions in [21] that the norms of $M_{p}^{(j)}$ satisfy $\left\|M_{p}^{(j)}\right\|_{\infty} \leq \lambda$ for all $j$. This inequality is a sufficient condition to ensure the existence of an invariant set for the prediction dynamics of $\left(z_{p}, \theta_{p}\right)$ in (31)-(32), which is the premise of the construction of terminal sets in Section 4.1.

In addition, $N_{v, p}$, the number of rows of $V_{p}$, represents the number of the tube facets. In general, the larger $N_{v, p}$ the designers choose, the higher dimension of the decision variable $\theta_{p}$ and the more linear inequality constraints are in the MPC optimization problem, thereby resulting in higher computational burden. However, it also implies the less conservative tube. Hence, in practice, the parameter $N_{v, p}$ can be tuned to balance the conservatism and the computational time.

To relax (21), (23), and (25) applied online, $M_{p}^{(j)}, M_{p}$, and $M_{c p}$ can be defined offline as the solutions of the corresponding optimization problems [13]

$$
\begin{aligned}
\left(M_{p}^{(j)}\right)_{i} & =\underset{\mathbf{m}^{T}}{\operatorname{argmin}}\left\{\mathbf{1}^{T} \mathbf{m} \mid \mathbf{m}^{T} V_{p}=\left(V_{p}\right)_{i}\left(\Psi_{p}^{0}+\Psi_{p}^{(j)}\right), \mathbf{m} \geq \mathbf{0}\right\}, \\
M_{p} & =\underset{\mathbf{m}^{T}}{\operatorname{argmin}}\left\{\mathbf{1}^{T} \mathbf{m} \mid \mathbf{m}^{T} V_{p}=\eta_{p}^{T}, \mathbf{m} \geq \mathbf{0}\right\}, \\
M_{c p} & =\underset{\mathbf{m}^{T}}{\operatorname{argmin}}\left\{\mathbf{1}^{T} \mathbf{m} \mid \mathbf{m}^{T} V_{p}=\mu_{c p}^{T}, \mathbf{m} \geq \mathbf{0}\right\},
\end{aligned}
$$

where $\mathbf{m} \in \mathbb{R}^{N_{v, p}}$ and the notation $(M)_{i}$ denotes the $i$ th row of matrix $M$. The linear programming problems (60)-(62) can be solved in polynomial time [22].

\subsection{Choice of Update Sequence}

Under the centralized algorithm, inter-subsystem communication must be performed at each time step and the total number of data transmissions is fixed. In comparison, for the proposed DSMPC algorithm, the flexibility in update sequence choice can be utilized to determine when data transmissions take place and how many data transmissions are required over the duration of a simulation. It implies that low levels of communication can be achieved through a predetermined update sequence by the designer. Furthermore, thanks to the tube methodology adopted to deal with probabilistic constraints, the non-optimizing subsystems at each time step remain inside their corresponding tubes, which ensures the control performance of the whole system with less frequent communication. The effect this flexibility in communication has on system performance has been explored in simulations in [10]. Accordingly, the DSMPC algorithm is particularly relevant for practical control problems in which communication is expensive or communication resources are limited (e.g., wireless communication via battery powered devices).

Based on the coupling structure of a system, the strategy for constructing an update sequence is discussed in the following. For the case of high degree of coupling, the optimizing subsystem is 
coupled with most subsystems or even all other subsystems. Take the worst-case as an example, i.e. all subsystems are coupled. If one chooses the update sequence such that each subsystem is impossible to optimize at two successive steps, for example $\{1,2,1,3,2,3, \ldots\}$, the new solution would be communicated to other subsystems at every time step. Instead, updating the same subsystem multiple times, for example $\{1,1,2,2,3,3, \ldots\}$, exchange of information is only required at alternative steps and the communication cost is decreased to half that of the first choice.

In another case that subsystems are less coupled, the update sequence can be chosen such that different subsystems optimize at successive time steps but can still ensure low frequency of communication. For that, all subsystems are divided into some coupling sets in which a coupled probabilistic constraint exists between these subsystems or there is a single subsystem decoupled to all other subsystems. The subsystems in different coupling sets optimize in a cyclical manner. For example, considering a system consisting of five subsystems and assuming that subsystems 1 and 2 and subsystems 4 and 5 are coupled via constraints, the coupling sets are defined as $S_{1}=\{1,2\}$, $S_{2}=\{3\}$, and $S_{3}=\{4,5\}$. In this example, choose the update sequence as $\{1,3,4,2,3,5, \ldots\}$ and therefore the new solution of an optimizing subsystem can be transmitted using the entire time interval after the optimization and before another subsystem in the same coupling set optimizes. Alternatively, it is possible for multiple subsystems from different coupling sets to optimize simultaneously at one time step, for example subsystems $1,3,4$ or subsystems $2,3,5$, which still preserves the properties of recursive feasibility and closed-loop stability.

\subsection{Computational Complexity}

We will discuss the computational complexity associated with the online computations involved in Algorithm 1.

The problem $\mathbf{P}_{p}^{D}\left(x_{p}(k) ; Z_{p}^{*}(k)\right)$ formulated in (47)-(54) is a convex optimization problem. In $\mathbf{P}_{p}^{D}\left(x_{p}(k) ; Z_{p}^{*}(k)\right)$, the cost function (47) is expressed as a quadratic function of $z_{p}(0 \mid k)$ and $\mathbf{c}_{p}(k)$. In addition, by choosing $V_{p}, M_{p}^{(j)}, M_{p}$, and $M_{c p}$ offline, the constraints (48)-(52) and the terminal constraint (54) are linear inequalities in decision variables and the terminal set $\chi_{p}^{0}$ is convex. Thus the optimization problem is convex, which can be efficiently implemented online. In particular, if we choose $\chi_{p}^{0}$ as some special forms, such as polyhedral robust invariant sets, the problem $\mathbf{P}_{p}^{D}\left(x_{p}(k) ; Z_{p}^{*}(k)\right)$ can be further cast as a quadratic program solved in polynomial time. The reduction in online computation time is achieved by determining parameters (e.g., $\beta_{p}^{i}$ and $\kappa_{c}^{i}$ ) that guarantee recursive feasibility through offline calculations.

To compare the computational requirements of Algorithm 1 and centralized SMPC, we focus on the analysis of the "size" per optimization including number of decision variables and number of constraints, which gives an indication of the computational complexity. Assume that there exist pair-wise couplings across the whole system. Hence, for the centralized algorithm, the number of coupled constraints is $N_{p}\left(N_{p}-1\right)$ which grows quadratically with $N_{p}$. Furthermore, the number of scalar decision variables is $N_{p}\left[\left(N_{1}+1\right) N_{v, p}+N_{x, p}+N_{u, p} N\right]$ which increases linearly with $N_{p}$. However, for Algorithm 1, the number of coupled constraints involving the optimizing subsystem $p$ is $\left(N_{p}-1\right)$ and the number of scalar decision variables is $\left(N_{1}+1\right) N_{v, p}+N_{x, p}+N_{u, p} N$ which is independent of $N_{p}$.

\section{NUMERICAL EXAMPLE}

The system to control comprises three subsystems, each with the same model parameters,

$$
\begin{aligned}
& A_{p}^{0}=\left[\begin{array}{cc}
1.6 & 1 \\
-0.5 & 1.2
\end{array}\right], B_{p}^{0}=\left[\begin{array}{l}
1 \\
1
\end{array}\right], \\
& A_{p}^{(1)}=\left[\begin{array}{cc}
0.05 & -0.15 \\
-0.01 & 0.01
\end{array}\right], B_{p}^{(1)}=\left[\begin{array}{c}
-0.05 \\
0.05
\end{array}\right], \\
& A_{p}^{(2)}=-A_{p}^{(1)}, B_{p}^{(2)}=-B_{p}^{(1)} .
\end{aligned}
$$



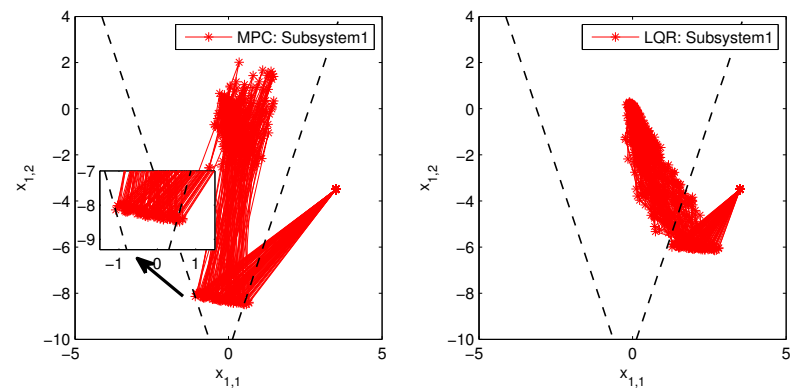

Figure 1. Closed-loop response of subsystem 1 under Algorithm 1 (left) and unconstrained optimal control (right) for 100 realizations of uncertainty.

Assume that $q_{p}^{(1)}(k)$ has a uniform distribution on $[0,1]$ and $q_{p}^{(2)}(k)=1-q_{p}^{(1)}(k)$. Each element of $w_{p}$ is truncated from a normal distribution with mean zero and variance $\frac{1}{10^{2}}$ and $w_{p}$ satisfies $\left\|w_{p}\right\|_{\infty} \leq 0.12$. Each subsystem is subject to two local probabilistic constraints and one coupled probabilistic constraint with parameters

$$
\begin{aligned}
& \eta_{p, 1}=[0.8-0.2]^{T}, h_{p, 1}=2.1, l_{p, 1}=0.8, \\
& \eta_{p, 2}=[-0.8-0.2]^{T}, h_{p, 2}=2.5, l_{p, 2}=0.8, \\
& \mu_{1 p}=[0.8-0.2]^{T}, b_{1}=5.5, \tilde{l}_{1}=0.8 .
\end{aligned}
$$

Choose $Q_{p}=\left[\begin{array}{ll}1 & 0.3\end{array}\right]^{T}\left[\begin{array}{ll}1 & 0.3\end{array}\right], R_{p}=0.1, N=4$, and $N_{1}=7$. With $N_{v, p}=8, V_{p}$ is chosen as

$$
V_{p}=\left[\begin{array}{cc}
-0.9997 & -0.0246 \\
-0.9898 & -0.1422 \\
0.9898 & -0.1422 \\
0.9997 & -0.0246 \\
0.9997 & 0.0246 \\
0.9898 & 0.1422 \\
-0.9898 & 0.1422 \\
-0.9997 & 0.0246
\end{array}\right]
$$

The fact that we choose $N_{v, p}=8$ is a tradeoff between conservatism and computational time. When $N_{v, p}=4$, the observed probabilities of constraint violations are very low although the computation speed is faster. But when $N_{v, p}>8$, there is no noticeable improvement in conservatism. Thus, $N_{v, p}=8$ is a reasonable choice. Solve (60)-(62) to obtain $M_{p}^{(j)}, M_{p}$, and $M_{c p}$, whose norms are $\left\|M_{p}^{(1)}\right\|_{\infty}=0.91,\left\|M_{p}^{(2)}\right\|_{\infty}=0.88,\left\|M_{p}\right\|_{\infty}=1.11$, and $\left\|M_{1 p}\right\|_{\infty}=1.11$. Choose $\tilde{\theta}_{p}=0.65$ which satisfies conditions (28)-(30) as required. The update sequence is set to be $\{1,2,3,1,2,3, \ldots\}$.

For $x_{1}(0)=[3.5-3.5]^{T}, x_{2}(0)=[3.4-3.45]^{T}$, and $x_{3}(0)=[3.5-3.25]^{T}$, Algorithm 1 and the unconstrained optimal control are applied to control the system in 1000 simulations. Fig. 1Fig. 4 show the evolution of $x_{p}(k)$ for each subsystem and $\sum_{p=1}^{3} x_{p}(k)$ under Algorithm 1 and the unconstrained optimal control according to 100 Monte Carlo simulations. The dashed lines denote state constraints.

With the unconstrained optimal control, local and coupled probabilistic constraints are violated at time $k=1$ for $100 \%$ of the uncertainty realizations. While under Algorithm 1, at time $k=1$, local constraints of subsystems 1,2 , and 3 are violated for $5.1 \%, 1 \%$, and $2.5 \%$ of the same 1000 uncertainty realizations respectively, and the observed probability of violating the state coupled constraint is $5.3 \%$.

The trajectories of three subsystems under Algorithm 1 for only one realization of uncertainty are shown in Fig. 5. From the simulation results, it is found that the system states converge to a neighborhood of the origin despite the action of both parameter uncertainty and disturbances. 

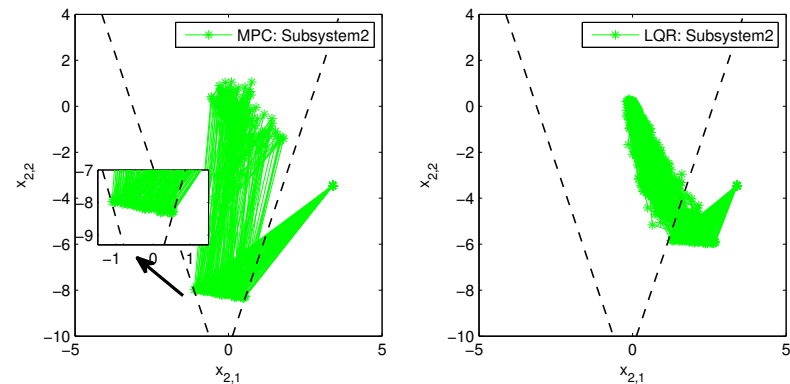

Figure 2. Closed-loop response of subsystem 2 under Algorithm 1 (left) and unconstrained optimal control (right) for 100 realizations of uncertainty.
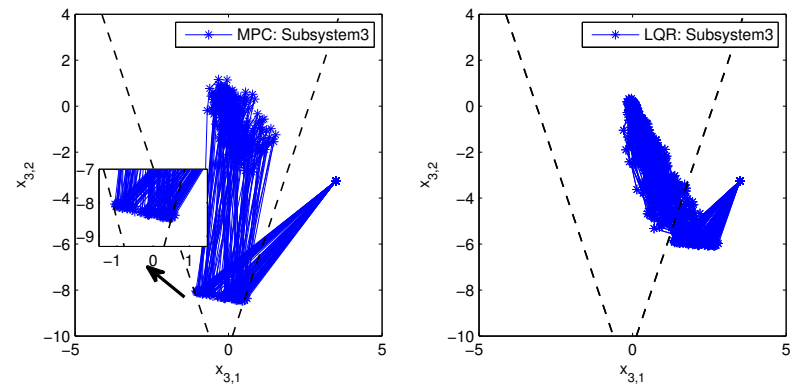

Figure 3. Closed-loop response of subsystem 3 under Algorithm 1 (left) and unconstrained optimal control (right) for 100 realizations of uncertainty.
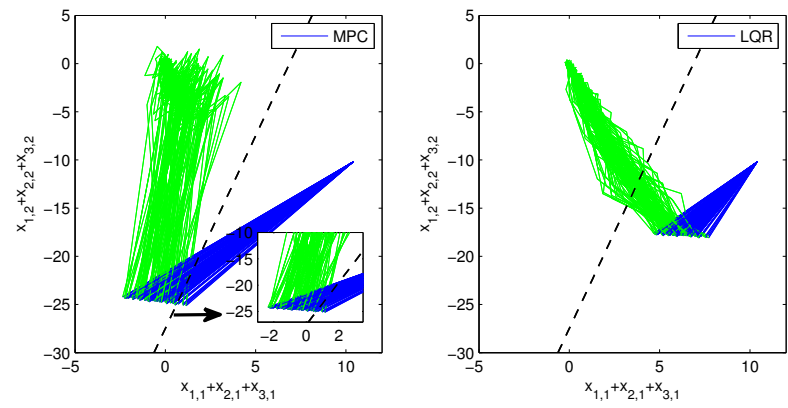

Figure 4. Evolution of coupled state $\sum_{p=1}^{3} x_{p}(k)$ under Algorithm 1 (left) and unconstrained optimal control (right) for 100 realizations of uncertainty of each subsystem.

Next we compare Algorithm 1 with the centralized SMPC in terms of the control performance and computational time. Define the performance index as

$$
J_{\text {perf }} \triangleq \sum_{k=0}^{T_{\text {run }}-1} \sum_{p=1}^{N_{p}}\left\|x_{p}(k)\right\|_{Q_{p}}^{2}+\left\|u_{p}(k)\right\|_{R_{p}}^{2},
$$

where $T_{\text {run }}$ is the simulation running time. By setting $T_{\text {run }}=10$, the control performance is 82.9543 for Algorithm 1 as compared with 70.3495 for the centralized SMPC. The average computation time per time step (over 1000 simulations) is 7.322 seconds for Algorithm 1 while it is 37.58 seconds for the centralized SMPC. 


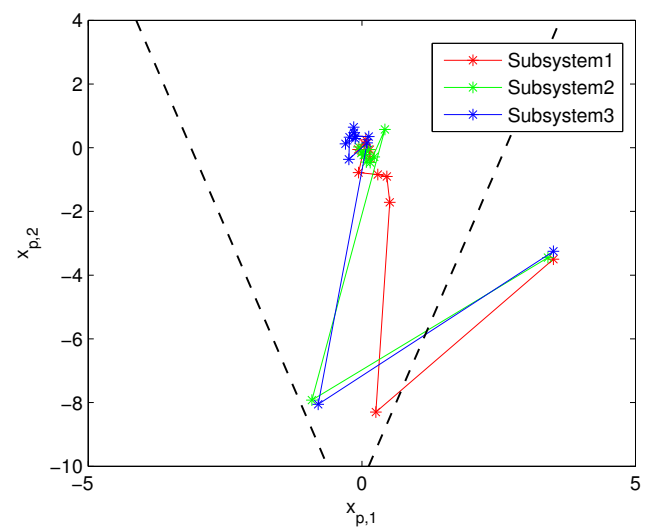

Figure 5. Closed-loop response of three subsystems under Algorithm 1 for only one realization of uncertainty of each subsystem.

\section{CONCLUSION}

Based on the tube invariant set theory, a DSMPC algorithm is proposed for a team of subsystems in the presence of both parameter uncertainty and disturbances. The uncertain state of the system is divided into two parts: one is constrained to lie in probabilistic tubes with no particular shape computed offline from the distribution of disturbances; the other is constrained to lie in polytopic tubes with facets of fixed orientation, and the volume of the tube is optimized online. By permitting only one subsystem to optimize locally at each time step, the new algorithm can ensure the satisfactions of coupled probabilistic constraints in a distributed manner, while achieving low communication costs and a flexibility in communication. The resulting optimization problem of DSMPC is a convex optimization problem, which can be solved efficiently. The proposed DSMPC algorithm guarantees recursive feasibility and stability for any choice of update sequence. A numerical example illustrates the efficacy of the algorithm.

In order to provide guidance for practical applications, our future research focus will be on the quantitative analysis of control performance of large-scale stochastic systems under different DMPC algorithms, particularly sequential DMPC [8, 10], synchronous DMPC [23, 24], and iterative DMPC $[25,26]$.

\section{APPENDIX}

Proof of Theorem 3.2. From (12), we have

$$
\begin{aligned}
V_{p} \zeta_{p}(i+1 \mid k)= & V_{p} \Psi_{p}(i \mid k) \zeta_{p}(i \mid k)+V_{p} \bar{\Psi}_{p}(i \mid k) z_{p}(i \mid k) \\
& +V_{p} \bar{B}_{p}(i \mid k) c_{p}(i \mid k)+V_{p} \bar{\Psi}_{p}(i \mid k) \varepsilon_{p}(i \mid k) .
\end{aligned}
$$

Applying Lemma 3.1 and using the facts that $\Psi_{p}(k)=\sum_{j=1}^{L}\left(\Psi_{p}^{0}+\Psi_{p}^{(j)}\right) q_{p}^{(j)}(k), \quad \Psi_{p}^{(j)}=$ $A_{p}^{(j)}+B_{p}^{(j)} K_{p}, \bar{\Psi}_{p}(k)=\sum_{j=1}^{L} \Psi_{p}^{(j)} q_{p}^{(j)}(k)$, and $\bar{B}_{p}(k)=\sum_{j=1}^{L} B_{p}^{(j)} q_{p}^{(j)}(k)$, we can obtain (20)(21).

Proof of Theorem 4.1. If $\theta_{p}(i \mid k) \in \mathcal{B}_{\tilde{\theta}_{p}}^{+}, i \in \mathbb{N}_{\geq N_{1}}$, it holds by (28) that $\max _{j}\left\|M_{p}^{(j)}\right\|_{\infty}\left\|\theta_{p}(i \mid k)\right\|_{\infty}+\max _{j}\left(\left\|v_{p, l}^{(j)}\right\|_{\infty}+\left\|\bar{d}_{p}^{(j)}\right\|_{\infty}\right) \leq \tilde{\theta}_{p} . \quad$ When $\quad z_{p}(i \mid k) \in \chi_{p}^{l}$, taking norms on both sides of (32) and applying the triangle inequality, we conclude that $\left\|\theta_{p}(i+1 \mid k)\right\|_{\infty} \leq \max _{j}\left\|M_{p}^{(j)}\right\|_{\infty}\left\|\theta_{p}(i \mid k)\right\|_{\infty}+\max _{j}\left(\left\|v_{p, l}^{(j)}\right\|_{\infty}+\left\|\bar{d}_{p}^{(j)}\right\|_{\infty}\right), \quad$ which $\quad$ yields $\left\|\theta_{p}(i+1 \mid k)\right\|_{\infty} \leq \tilde{\theta}_{p}$. Hence, $\mathcal{B}_{\tilde{\theta}_{p}}^{+}$is an invariant set of (32). This together with the assumption of $\chi_{p}^{l}$ ensures that $\chi_{p}^{l} \times \mathcal{B}_{\tilde{\theta}_{p}}^{+}$is an invariant set for the dynamics of $\left(z_{p}, \theta_{p}\right)$ given by (31)-(32). 
The condition (29) is equivalent to $\left\|M_{p}\right\|_{\infty} \tilde{\theta}_{p}+\bar{\beta}_{p}+\left\|f_{p, l}\right\|_{\infty} \leq h_{p}$, which implies $\left\|M_{p}\right\|_{\infty}\left\|\theta_{p}(i \mid k)\right\|_{\infty}+\bar{\beta}_{p}+\left\|f_{p, l}\right\|_{\infty} \leq h_{p}$ if $\quad \theta_{p}(i \mid k) \in \mathcal{B}_{\tilde{\theta}_{p}}^{+}$. Furthermore, when $z_{p}(i \mid k) \in \chi_{p}^{l}$, the definitions of $f_{p, l}$ and $\bar{\beta}_{p}$ guarantee that $\left\|M_{p} \theta_{p}(i \mid k)+\beta_{p}^{i}+\eta_{p}^{T} z_{p}(i \mid k)\right\|_{\infty} \leq h_{p}$. Hence, if $\left(z_{p}(i \mid k), \theta_{p}(i \mid k)\right) \in \chi_{p}^{l} \times \mathcal{B}_{\tilde{\theta}_{p}}^{+}, i \in \mathbb{N}_{\geq N_{1}}$, then (23) holds.

With similar arguments, if $\left(z_{p}(i \mid k), \theta_{p}(i \mid k)\right) \in \chi_{p}^{l} \times \mathcal{B}_{\tilde{\theta}_{p}}^{+}$and $\tilde{\theta}_{p}$ satisfies (30) for each subsystem $p$, then (25) also holds.

Proof of Lemma 5.1. Pre- and post- multiplication of (45) by $\left[z_{p}^{T}(i \mid k) c_{p}^{T}(i \mid k)\right]$ and $\left[z_{p}^{T}(i \mid k) c_{p}^{T}(i \mid k)\right]^{T}$ yields

$$
\begin{aligned}
& \left\|\left[\begin{array}{c}
z_{p}(i \mid k) \\
c_{p}(i \mid k)
\end{array}\right]\right\|_{W_{p}}^{2}-\left\|\left[\begin{array}{c}
z_{p}(i+1 \mid k) \\
c_{p}(i+1 \mid k)
\end{array}\right]\right\|_{W_{p}}^{2} \\
= & \left\|z_{p}(i \mid k)\right\|_{Q_{p}}^{2}+\left\|K_{p} z_{p}(i \mid k)+c_{p}(i \mid k)\right\|_{R_{p}}^{2} .
\end{aligned}
$$

Summing over $i \in \mathbb{N}$ will lead to (46).

Proof of Theorem 5.1. To prove recursive feasibility in (i), we must show the existence of feasible solutions to $\mathbf{P}_{p}^{D}\left(x_{p}(k) ; Z_{p}^{*}(k)\right)$ at every time step, given an initial feasible solution for each subsystem $p \in \mathcal{P}$. Supposing that at time $k_{0}$, a feasible solution to $\mathbf{P}_{p}^{D}\left(x_{p}\left(k_{0}\right) ; Z_{p}^{*}\left(k_{0}\right)\right)$ for each subsystem $p \in \mathcal{P}$ is given by $\left\{\mathbf{c}_{p}\left(k_{0}\right), z_{p}\left(0 \mid k_{0}\right), \theta_{p}\left(0 \mid k_{0}\right), \theta_{p}\left(1 \mid k_{0}\right), \ldots, \theta_{p}\left(N_{1} \mid k_{0}\right)\right\}$, we will prove that the candidate solution

$$
\begin{aligned}
& \left\{T_{p} \mathbf{c}_{p}\left(k_{0}\right), z_{p}\left(1 \mid k_{0}\right)+w_{p}\left(k_{0}\right), \theta_{p}\left(1 \mid k_{0}\right), \ldots, \theta_{p}\left(N_{1} \mid k_{0}\right),\right. \\
& \left.\quad \max _{j}\left\{M_{p}^{(j)} \theta_{p}\left(N_{1} \mid k_{0}\right)+V_{p} \Psi_{p}^{(j)} z_{p}\left(N_{1} \mid k_{0}\right)+d_{p}^{(j)}\left(N_{1}\right)\right\}\right\}
\end{aligned}
$$

is a feasible solution to $\mathbf{P}_{p}^{D}\left(x_{p}\left(k_{0}+1\right) ; Z_{p}^{*}\left(k_{0}+1\right)\right)$, i.e., (63) satisfies constraints (48)-(54) at time $k_{0}+1$.

Firstly, for (48), since $V_{p} \zeta_{p}\left(1 \mid k_{0}\right) \leq \theta_{p}\left(1 \mid k_{0}\right)$ at time $k_{0}$ by assumption and $\zeta_{p}\left(1 \mid k_{0}\right)=x_{p}\left(k_{0}+\right.$ $1)-z_{p}\left(1 \mid k_{0}\right)-w_{p}\left(k_{0}\right)$ by the definition of $\zeta_{p}$, (48) is satisfied at time $k_{0}+1$ for the choices of $z_{p}\left(0 \mid k_{0}+1\right)=z_{p}\left(1 \mid k_{0}\right)+w_{p}\left(k_{0}\right)$ and $\theta_{p}\left(0 \mid k_{0}+1\right)=\theta_{p}\left(1 \mid k_{0}\right)$ in (63).

Next, the nominal system equation (49) is satisfied at time $k_{0}+1$ directly. For $i \in \mathbb{N}_{\leq N_{1}-2}$, the satisfaction of constraints (50)-(51) at time $k_{0}+1$ follows directly from the assumption. For $i=N_{1}-1$, by taking $\theta_{p}\left(N_{1}-1 \mid k_{0}+1\right)=\theta_{p}\left(N_{1} \mid k_{0}\right)$ and $z_{p}\left(0 \mid k_{0}+1\right)=z_{p}\left(1 \mid k_{0}\right)+w_{p}\left(k_{0}\right)$ in (63) and using the fact that $d_{p}^{(j)}\left(N_{1}\right) \geq V_{p} \Psi_{p}^{(j)}\left(\Psi_{p}^{0}\right)^{N_{1}-1} w_{p}\left(k_{0}\right)+d_{p}^{(j)}\left(N_{1}-1\right)$, it holds that

$$
\begin{aligned}
& M_{p}^{(j)} \theta_{p}\left(N_{1}-1 \mid k_{0}+1\right)+V_{p} \Psi_{p}^{(j)} z_{p}\left(N_{1}-1 \mid k_{0}+1\right)+V_{p} B_{p}^{(j)} c_{p}\left(N_{1}-1 \mid k_{0}+1\right) \\
& +d_{p}^{(j)}\left(N_{1}-1\right) \leq \max _{j}\left\{M_{p}^{(j)} \theta_{p}\left(N_{1} \mid k_{0}\right)+V_{p} \Psi_{p}^{(j)} z_{p}\left(N_{1} \mid k_{0}\right)+d_{p}^{(j)}\left(N_{1}\right)\right\},
\end{aligned}
$$

which implies that constraint (50) is also satisfied at time $k_{0}+1$ for $i=N_{1}-1$. Furthermore, by Theorem 4.1 and the assumption that $\left(z_{p}\left(N_{1} \mid k_{0}\right), \theta_{p}\left(N_{1} \mid k_{0}\right)\right) \in \chi_{p}^{N_{1}-N} \times \mathcal{B}_{\tilde{\theta}_{p}}^{+}$, it yields that

$$
M_{p} \theta_{p}\left(N_{1} \mid k_{0}\right)+\beta_{p}^{N_{1}}+\eta_{p}^{T} z_{p}\left(N_{1} \mid k_{0}\right) \leq h_{p} .
$$

Again taking $\theta_{p}\left(N_{1}-1 \mid k_{0}+1\right)=\theta_{p}\left(N_{1} \mid k_{0}\right)$ and $z_{p}\left(0 \mid k_{0}+1\right)=z_{p}\left(1 \mid k_{0}\right)+w_{p}\left(k_{0}\right)$ in (63) and utilizing the inequality $\beta_{p}^{N_{1}} \geq \beta_{p}^{N_{1}-1}+\eta_{p}^{T}\left(\Psi_{p}^{0}\right)^{N_{1}-1} w_{p}\left(k_{0}\right)$, we obtain

$$
M_{p} \theta_{p}\left(N_{1}-1 \mid k_{0}+1\right)+\beta_{p}^{N_{1}-1}+\eta_{p}^{T} H_{p}^{N_{1}-1} \mathbf{c}_{p}\left(k_{0}+1\right)+\eta_{p}^{T}\left(\Psi_{p}^{0}\right)^{N_{1}-1} z_{p}\left(0 \mid k_{0}+1\right) \leq h_{p},
$$

and therefore constraint (51) is also satisfied at time $k_{0}+1$ for $i=N_{1}-1$.

As for (52), by calculating the values of $\mathbf{c}_{q}^{*}\left(k_{0}+1\right), z_{q}^{*}\left(0 \mid k_{0}+1\right)$, and $\theta_{q}^{*}\left(i \mid k_{0}+1\right)$ according to (55)-(58) and using the facts that

$$
\begin{gathered}
\Xi_{c q}^{*}\left(i \mid k_{0}+1\right)=\Xi_{c q}^{*}\left(1+i \mid k_{0}\right)+\left|\mu_{c q}^{T}\left(\Psi_{q}^{0}\right)^{i}\right| \alpha_{q}, \\
\kappa_{c}^{i}+\mu_{c p}^{T}\left(\Psi_{p}^{0}\right)^{i} w_{p}\left(k_{0}\right)+\sum_{q \in \mathcal{P}_{c} \backslash\{p\}}\left|\mu_{c q}^{T}\left(\Psi_{q}^{0}\right)^{i}\right| \alpha_{q} \leq \kappa_{c}^{i+1},
\end{gathered}
$$


it holds that (52) is satisfied at time $k_{0}+1$ for $i \in \mathbb{N}_{\left[1, N_{1}-2\right]}$. In addition, since the terminal constraints $\left(z_{p}\left(N_{1} \mid k_{0}\right), \theta_{p}\left(N_{1} \mid k_{0}\right)\right) \in \chi_{p}^{N_{1}-N} \times \mathcal{B}_{\tilde{\theta}_{p}}^{+}$and $\left(z_{q}^{*}\left(N_{1} \mid k_{0}\right), \theta_{q}^{*}\left(N_{1} \mid k_{0}\right)\right) \in \chi_{q}^{N_{1}-N} \times \mathcal{B}_{\tilde{\theta}_{q}}^{+}$ are satisfied at time $k_{0}$, and

$$
\Xi_{c q}^{*}\left(N_{1}-1 \mid k_{0}+1\right)=\Xi_{c q}^{*}\left(N_{1} \mid k_{0}\right)+\left|\mu_{c q}^{T}\left(\Psi_{q}^{0}\right)^{N_{1}-1}\right| \alpha_{q},
$$

it follows from Theorem 4.1 that (25) holds, which further leads to the satisfaction of (52) at time $k_{0}+1$ for $i=N_{1}-1$. Thus, we can conclude that the candidate solution (63) is also feasible to (52) at time $k_{0}+1$.

Finally, it remains to show that (63) satisfies the terminal constraints (53)-(54) at time $k_{0}+1$. With the choice $z_{p}\left(0 \mid k_{0}+1\right)=z_{p}\left(1 \mid k_{0}\right)+w_{p}\left(k_{0}\right)$ in (63), it follows from (31) that $z_{p}\left(N \mid k_{0}+1\right)=$ $\Psi_{p}^{0} z_{p}\left(N \mid k_{0}\right)+\left(\Psi_{p}^{0}\right)^{N} w_{p}\left(k_{0}\right)$, which leads to $z_{p}\left(N \mid k_{0}+1\right) \in \chi_{p}^{0}$ in (53). In addition, since $\mathcal{B}_{\tilde{\theta}_{p}}^{+}$is a invariant set for (32) and $\left\|\theta_{p}\left(N_{1} \mid k_{0}\right)\right\|_{\infty} \leq \tilde{\theta}_{p}$ by assumption, we can conclude that

$$
\begin{aligned}
& \left\|\theta_{p}\left(N_{1}+1 \mid k_{0}\right)\right\|_{\infty} \\
= & \left\|\max _{j}\left\{M_{p}^{(j)} \theta_{p}\left(N_{1} \mid k_{0}\right)+V_{p} \Psi_{p}^{(j)} z_{p}\left(N_{1} \mid k_{0}\right)+d_{p}^{(j)}\left(N_{1}\right)\right\}\right\|_{\infty} \\
\leq & \tilde{\theta}_{p} .
\end{aligned}
$$

So, by choosing $\theta_{p}\left(N_{1} \mid k_{0}+1\right)=\max _{j}\left\{M_{p}^{(j)} \theta_{p}\left(N_{1} \mid k_{0}\right)+V_{p} \Psi_{p}^{(j)} z_{p}\left(N_{1} \mid k_{0}\right)+d_{p}^{(j)}\left(N_{1}\right)\right\}$ in (63), (54) holds at time $k_{0}+1$.

Hence (63) is a feasible solution to $\mathbf{P}_{p}^{D}\left(x_{p}\left(k_{0}+1\right) ; Z_{p}^{*}\left(k_{0}+1\right)\right)$. By recursion, recursive feasibility of $\mathbf{P}_{p}^{D}\left(x_{p}(k) ; Z_{p}^{*}(k)\right), k=k_{0}+1, k_{0}+2, \ldots$, follows immediately, thereby completing the proof of (i).

For (ii), since the constraints (51) and (52) incorporated in $\mathbf{P}_{p}^{D}\left(x_{p}(k) ; Z_{p}^{*}(k)\right)$ and recursive feasibility of $\mathbf{P}_{p}^{D}\left(x_{p}(k) ; Z_{p}^{*}(k)\right)$ obtained in (i), the satisfactions of (13) and (14) follow from Theorem 3.3, thereby ensuring the satisfactions of probabilistic constraints (3) and (4) by Theorem 3.1 .

Proof of Theorem 5.2. Due to recursive feasibility in Theorem 5.1 (i) and the assumption that $u_{p}(k)=K_{p} x_{p}(k)$ is the unconstrained optimal control law, $\left\{c_{p}(0), c_{p}(1), \ldots\right\}$ is in $l^{2}$, which means that $c_{p}(k)$ converges to zero as $k \rightarrow \infty$. Hence, the MPC control law $u_{p}(k)=K_{p} x_{p}(k)+c_{p}(k)$ converges asymptotically to the unconstrained optimal control law $u_{p}(k)=K_{p} x_{p}(k)$ which can stabilize the subsystem by assumption, and meanwhile the state $x_{p}(k)$ converges asymptotically to a set of states in which the constraints (8)-(9) are satisfied by applying $u_{p}(k)=K_{p} x_{p}(k)$.

\section{ACKNOWLEDGEMENT}

This work was supported by the Beijing Natural Science Foundation under Grant 416100, the National Natural Science Foundation of China under Grant 61603041, Grant 61225015, Grant 61105092 and Grant 61422102, Foundation for Innovative Research Groups of the National Natural Science Foundation of China under Grant 61321002, and Young Elite Scientists Sponsorship Program by CAST under Grant 2016 QNRC001.

\section{REFERENCES}

1. Ding B, Xie L, Cai W. Distributed model predictive control for constrained linear systems. International Journal of Robust and Nonlinear Control 2010; 20(11):1285-1298.

2. Müller M, Reble M, Allgöwer F. Cooperative control of dynamically decoupled systems via distributed model predictive control. International Journal of Robust and Nonlinear Control 2012; 22(12):1376-1397.

3. Maestre J, Negenborn R. Distributed model predictive control made easy. Springer: Netherlands, 2014.

4. Cannon M, Kouvaritakis B, Wu X. Probabilistic constrained MPC for multiplicative and additive stochastic uncertainty. IEEE Transactions on Automatic Control 2009; 54(7):1626-1632.

5. Li J, Li D, Xi Y, Zhang P. Stochastic output-feedback model predictive control for systems with multiplicative and additive uncertainty. Proceedings of the 34th Chinese Control Conference (CCC), 2015; 4179-4184.

6. Cheng Q, Cannon M, Kouvaritakis B, Evans M. Stochastic MPC for systems with both multiplicative and additive disturbances. Proceedings of the 19th IFAC World Congress, 2014; 2291-2296. 
7. Kouvaritakis B, Rossiter J, Schuurmans J. Efficient robust predictive control. IEEE Transactions on Automatic Control 2000; 45(8):1545-1549.

8. Richards A, How J. Robust distributed model predictive control. International Journal of Control 2007; 80(9):15171531.

9. Mayne D, Seron M, Raković S. Robust model predictive control of constrained linear systems with bounded disturbances. Automatica 2005; 41(2):219-224.

10. Trodden P, Richards A. Distributed model predictive control of linear systems with persistent disturbances. International Journal of Control 2010; 83(8):1653-1663.

11. Dai L, Xia Y, Gao Y, Kouvaritakis B, Cannon M. Cooperative distributed stochastic MPC of linear systems with state estimation and coupled probabilistic constraints. Automatica 2015; 61:89-96.

12. Dai L, Xia Y, Gao Y. Distributed MPC of linear systems with stochastic parametric uncertainties and coupled probabilistic constraints. SIAM Journal on Control and Optimization 2015; 53(6):3411-3431.

13. Fleming J, Kouvaritakis B, Cannon M. Regions of attraction and recursive feasibility in robust MPC. Proceedings of the 21st Mediterranean Conference on Control \& Automation, 2013; 801-806.

14. Fleming J, Kouvaritakis B, Cannon M. Robust tube MPC for linear systems with multiplicative uncertainty. IEEE Transactions on Automatic Control 2015; 60(4):1087-1092.

15. Kouvaritakis B, Cannon M, Raković S, Cheng Q. Explicit use of probabilistic distributions in linear predictive control. Automatica 2010; 46(10):1719-1724.

16. Camponogara E, Jia D, Krogh B, Talukdar S. Distributed model predictive control. IEEE Control Systems Magazine 2002; 22(1):44-52.

17. Maestre J, Peña D de la, Camacho E. Distributed MPC based on agent negotiation, in Distributed model predictive control made easy, Maestre J, Negenborn R, Eds. Springer: Netherlands, 2014: 465-477.

18. Liu J, Peña D de la, Christofides P. Lyapunov-based distributed MPC schemes: Sequential and iterative approaches, in Distributed model predictive control made easy, Maestre J, Negenborn R, Eds. Springer: Netherlands, 2014: 479494.

19. Cheng Q. Robust \& stochastic model predictive control. Doctoral dissertation, University of Oxford, 2012.

20. Blanchini F, Miani S. Set-theoretic methods in control. Springer: Boston, 2007.

21. Bitsoris G. On the positive invariance of polyhedral sets of discrete-time linear systems. System and Control Letters 1988; 11(3):243-248.

22. RinnooyKan A, Telgen J. The complexity of linear programming. Statistica Neerlandica 1981; 35(2):91-107.

23. Dunbar W. Distributed receding horizon control of dynamically coupled nonlinear systems. IEEE Transactions on Automatic Control 2007; 52(7):1249-1263.

24. Gao Y, Dai L, Xia Y, Liu Y. Distributed model predictive control for consensus of nonlinear second-order multi-agent systems. International Journal of Robust and Nonlinear Control 2017; 27(5):830-842.

25. Ferramosca A, Limn D, Alvarado I, Camacho E. Cooperative distributed MPC for tracking. Automatica 2013; 49(4):906-914

26. Stewart B, Wright S, Rawlings J. Cooperative distributed model predictive control for nonlinear systems. Journal of Process Control 2011; 21(5):698-704. 